\title{
Algebraic construction of a third order difference approximation for fractional derivatives and applications
}

\author{
HM Nasir $^{1} \quad$ K Nafa $^{2}$
}

Received 4 November 2017; revised 09 July 2018

\begin{abstract}
Finite difference approximations for fractional derivatives based on Grünwald formula are well known to be of first order accuracy, but display unstable solutions with known numerical methods. The shifted form of the Grünwald approximation removes this instability and keeps the same first order accuracy. Higher order approximations have been obtained by convex combinations of various shifted Grünwald approximations. Recently, a second order shifted Grünwald type approximation was constructed algebraically through a generating function. In this paper, we derive a new third order approximation from this second order approximation by preconditioning the fractional differential operator. This approximation is used with Crank-Nicolson
\end{abstract}

DoI:10.21914/anziamj.v59i0.12592, (C) Austral. Mathematical Soc. 2018. Published November 9, 2018, as part of the Proceedings of the 13th Biennial Engineering Mathematics and Applications Conference. ISSN 1445-8810. (Print two pages per sheet of paper.) Copies of this article must not be made otherwise available on the internet; instead link directly to the DOI for this article. 
numerical scheme to approximate the solutions of space-fractional diffusion equations by the same preconditioning. Stability and convergence of the numerical scheme are analysed, supported by numerical results showing third order convergence.

Subject class: 34A08;34D20;65L07;65L12;65L20

Keywords: Grünwald approximation; Generating function; Fractional diffusion equation; Crank-Nicolson scheme; Stability and convergence.

\section{Contents}

1 Introduction

C255

2 Generalised Grünwald approximations

$\mathrm{C} 258$ 2.1 A new third order approximation ......... C260

3 Fractional diffusion equation

C261 3.1 Stability and convergence ............. C262

4 Numerical results

C265

5 Conclusion

C267

References

C268

\section{Introduction}

Fractional integral and fractional derivative (FD) are extensions of the integer order integrals and derivatives to a real or complex order. FD is suitable to describe anomalous transport in an external field derived from the continuous time random walk [2], resulting in a fractional diffusion equation (FDE). 
The FDE involves fractional derivatives either in time, in space or in both variables.

Among various definitions of FD, the Grünwald-Letnikov (G-L) definition and its Grünwald approximation are commonly used in numerical computations. We start with the left(-) and right(+) G-L definitions with shift $r$ for FD of a function $f(x)$ defined in $\mathbb{R}$.

$$
D_{x \mp, r}^{\alpha} f(x)=\lim _{h \rightarrow 0} \frac{1}{h^{\alpha}} \sum_{k=0}^{\infty} g_{k}^{(\alpha)} f(x \mp(k-r) h),
$$

where $g_{k}^{(\alpha)}=(-1)^{k}\left(\begin{array}{l}\alpha \\ k\end{array}\right)=(-1)^{k} \frac{\Gamma(\alpha+1)}{\Gamma(\alpha+1-k) k !}$ with gamma function $\Gamma(\cdot)$. The classical G-L definitions have no shift and hence are given by (1) with $r=0$. The coefficients $g_{k}^{(\alpha)}$ are the coefficients of the binomial expansion of the generating function $W_{1}(z)=(1-z)^{\alpha}$.

For a fixed $h>0$, the Grünwald approximations are obtained by simply dropping the limit in the G-L definition given in equation (1).

When $f(x)$ is defined in an interval $[a, b]$, it is zero-extended outside the interval to adopt these definitions and approximations. The upper limit infinity of the sum in (1) is then restricted to $N+r$, where $N=[(x-a) / h]$ and $N=[(b-x) / h]$ for the left and right approximations respectively, with [y] denoting the integer part of $y$. The addition of $r$ in the upper limit is to cover all the discrete points from $x$ to the relevant boundary of the interval.

Use of this approximation in FDEs results in unstable solutions when Euler, implicit Euler and even Crank-Nicolson (C-N) numerical schemes are employed. The last two schemes are well known for unconditional stability in classical diffusion problems. Meerchaert et al. [5] showed that the approximations with shift $r=1$ removes this instability, but the order of accuracy remains equal to one.

Earlier, Lubich [4] obtained higher order approximations for FD up to order six based on backward multi-step methods for initial value problems. Experiments 
show that these approximations also suffer the same instability as in the first order case. When a shifted form for these approximations is used, though the stability is restored, the orders of the approximations drop to one for all the six cases making them unusable for higher order numerical schemes [7].

The search for higher order approximations with shifts has attracted many researchers recently. Nasir et al. [6] obtained a second order approximation through a non-integer shift in the Grünwald approximation, displaying super convergence. Convex combinations of various shifts of the shifted Grünwald approximation were used to obtain higher order approximations in Chinese schools $[8,9,10,11]$. Baeumer et al. [1] considered convex combinations of shifted Grünwald approximation with different step sizes and extrapolated to obtain higher order approximations and analysed their stability and order of convergence based on the smoothness of an approximated function. All these higher order approximations are obtained by manipulating the first order shifted Grünwald approximation only.

A generalisation of the concept of shifted Grünwald approximation by identifying it with a generating function has previously been proposed in [7], and a generating function for a second order approximation with shift was constructed, analysed and used to solve fractional diffusion problems.

In this paper, firstly, we construct a third order shifted Grünwald type approximation from this second order generating function (Section 2). Secondly, the $\mathrm{C}-\mathrm{N}$ scheme and its stability for sufficiently smooth functions are analysed (Section 3). Thirdly, we test our scheme for examples with sufficiently smooth solutions (Section 4). We also check the effect of smoothness of the approximated function on the order of convergence to see if the results in [1] are achieved in our case as well. 


\section{Generalised Grünwald approximations}

We derived some higher order Grünwald type approximations that retain their higher orders with non-zero shifts [7]. Among them, the second order approximation was shown to be reliable for stability and approximation order. We give the definitions and theorems used to construct higher order approximations in [7].

For a sufficiently smooth function $f(x)$ and generic weights $w_{k, r}^{(\alpha)}$, define the left and right Grünwald type operators with shift $r$ as

$$
\Delta_{h \mp, r}^{\alpha} f(x)=\frac{1}{h^{\alpha}} \sum_{k=0}^{\infty} w_{k, r}^{(\alpha)} f(x \mp(k-r) h) .
$$

Definition 1. A sequence $\left\{w_{k, r}^{(\alpha)}\right\}$ of real numbers, or its generating function $W_{\mathrm{r}}(z)=\sum_{\mathrm{k}=0}^{\infty} w_{\mathrm{k}, \mathrm{r}}^{(\alpha)} z^{\mathrm{k}}$, is said to approximate the left and right fractional derivatives $D_{x \mp}^{\alpha}$ at $x$ with shift $r$ in the sense of Grünwald if

$$
D_{x \mp}^{\alpha} f(x)=\lim _{h \rightarrow 0} \Delta_{h \mp, r}^{\alpha} f(x) .
$$

The sequence is said to approximate $D_{x \mp}^{\alpha}$ with order $p \geqslant 1$ and shift $r$ if

$$
D_{x \mp}^{\alpha} f(x)=\Delta_{h \mp, r}^{\alpha} f(x)+O\left(h^{p}\right) .
$$

In the latter case, we denote the generating function as $W_{p, r}(z)$ and the approximation operator as $\Delta_{\mathrm{h} \mp, \mathrm{r}, \mathrm{p}}^{\alpha} \mathrm{f}(\mathrm{x})$.

An equivalent characterisation of the approximating generator $W_{p, r}(z)$ with order $p \geqslant 1$ and shift $r$ is given by the following Theorem [7].

Theorem 2. Let $\mathrm{n}=[\alpha]+1, \mathrm{~m}$ a non-negative integer, $\mathrm{f}(\mathrm{x}) \in \mathrm{C}^{\mathrm{m}+\mathrm{n}+1}(\mathbb{R})$ and $\mathrm{D}_{\mathrm{x}}^{\mathrm{k}} \mathrm{f}(\mathrm{x})=\mathrm{d}^{\mathrm{k}} / \mathrm{d} \mathrm{x}^{\mathrm{k}} \mathrm{f}(\mathrm{x}) \in \mathrm{L}_{1}(\mathbb{R})$ for $0 \leqslant \mathrm{k} \leqslant \mathrm{m}+\mathrm{n}+1$. Then, the generating function $\mathrm{W}_{\mathrm{p}, \mathrm{r}}(z)$ approximates the left and right fractional differential 
operator $\mathrm{D}_{\mathrm{x} \mp}^{\alpha}$ with order $\mathrm{p}$ and shift $\mathrm{r}, 1 \leqslant \mathrm{p} \leqslant \mathrm{m}$, if and only if

$$
\mathrm{G}_{\mathrm{r}}(z):=\frac{1}{z^{\alpha}} W_{p, r}\left(e^{-z}\right) e^{r z}
$$

is analytic in a neighbourhood $|z| \leqslant \mathrm{R}$ for some $\mathrm{R}>1$ and

$$
\mathrm{G}_{\mathrm{r}}(z)=1+\mathrm{O}\left(z^{\mathfrak{p}}\right) \text {. }
$$

Moreover, if $\mathrm{G}_{\mathrm{r}}(z)=1+\sum_{\mathrm{l}=\mathrm{p}}^{\infty} \mathrm{a}_{\mathrm{l}}(\mathrm{r}) z^{\mathrm{l}}$, we have

$$
\Delta_{h \mp, r, p}^{\alpha} f(x)=D_{x \mp}^{\alpha} f(x)+h^{p} a_{p} D_{x \mp}^{\alpha+p} f(x)+\cdots+O\left(h^{m}\right) .
$$

An immediate consequence of this is the following consistency condition.

Corollary 3. [7] If the generating function $\mathrm{W}_{\mathrm{r}}(z)$ approximates the fractional differential operators, then $\mathrm{W}_{\mathrm{r}}(1)=0$.

In [7], we constructed algebraically some higher order approximating generating functions of the form $W_{p, r}(z)=\left(\beta_{0}+\beta_{1} z+\cdots+\beta_{p} z^{p}\right)^{\alpha}$ using Theorem 2. This was achieved from the Taylor series expansion $G_{r}(z)=\sum_{l=0}^{\infty} a_{l} z^{l}$ by setting the constrains $a_{0}=1, a_{l}=0,1 \leqslant l \leqslant p-1$, along with the consistency condition $\beta_{0}+\beta_{1}+\cdots+\beta_{p}=0$ from Corollary 3 , and solving the resulting linear system for $\beta_{j}$.

An order 2 approximating generating function $W_{2, \mathrm{r}}(z)=\left(\beta_{0}+\beta_{1} z+\beta_{2} z^{2}\right)^{\alpha}$ was constructed yielding $\beta_{0}=\frac{3}{2}-\lambda, \quad \beta_{1}=-2+2 \lambda \quad$ and $\quad \beta_{2}=\frac{1}{2}-\lambda$, where $\lambda=r / \alpha$. We then have from Theorem 2, equation (5),

$$
\Delta_{h \mp, r, 2}^{\alpha} f(x)=D_{x \mp}^{\alpha} f(x)+O\left(h^{2}\right) .
$$

Note that when there is no shift, $W_{2, \mathrm{r}}(z)$ becomes the second order generator $W_{2,0}(z)=\left(3 / 2-2 z+z^{2} / 2\right)^{\alpha}$ given in Lubich [4]. This is true for all the generators $W_{p, r}(z), 1 \leqslant p \leqslant 6$. Hence, our higher order generators are extensions of the Lubich generators with shifts. 


\subsection{A new third order approximation}

Our main result here is that the operator $\Delta_{h \mp, r, 2}^{\alpha}$ in (6) can be used to approximate a preconditioned operator $\mathrm{P}_{x} \mathrm{D}_{x \mp}^{\alpha}$ with order 3 accuracy by retaining the term of $h^{2}$ in (6). In fact, we have from (6),

$$
\begin{aligned}
\Delta_{h \mp, r, 2}^{\alpha} f(x) & =D_{x \mp}^{\alpha} f(x)+a_{2} h^{2} D_{x \mp}^{2+\alpha} f(x)+O\left(h^{3}\right), \\
& =\left(I+a_{2} h^{2} D_{x}^{2}\right) D_{x \mp}^{\alpha} f(x)+O\left(h^{3}\right), \\
& =P_{x} D_{x \mp}^{\alpha} f(x)+O\left(h^{3}\right),
\end{aligned}
$$

where $P_{x}=\left(I+h^{2} a_{2} D_{x}^{2}\right)$ and I denotes the identity operator. The coefficient $a_{2}$ in this case is given by $a_{2}=-\frac{\alpha}{3}+r-\frac{r^{2}}{2 \alpha}$.

When $r=1$, the differential operator $D_{x}^{2}$ is approximated by the central difference operator $\delta_{h}^{2} f(x)=\frac{1}{h^{2}}(f(x-h)-2 f(x)+f(x+h))$ with $D_{x}^{2} f(x)=$ $\delta_{h}^{2} f(x)+O\left(h^{2}\right)$. From this, an approximation for the operator $P_{x}$ is obtained as

$$
\begin{aligned}
P_{x} f(x) & =\left(I+a_{2} h^{2} D_{x}^{2}\right) f(x)=\left(I+a_{2} h^{2}\left(\delta_{h}^{2}+O\left(h^{2}\right)\right) f(x)\right. \\
& =\left(I+a_{2} h^{2} \delta_{h}^{2}\right) f(x)+O\left(h^{4}\right) \\
& =P_{h} f(x)+O\left(h^{4}\right)
\end{aligned}
$$

where

$$
P_{h}=\left(I+a_{2} h^{2} \delta_{h}^{2}\right)
$$

We, therefore, have from (7) and (8),

$$
\begin{aligned}
\Delta_{h \mp, 1,2}^{\alpha} f(x) & =\left(P_{h}+O\left(h^{4}\right)\right) D_{x \mp}^{\alpha} f(x)+O\left(h^{3}\right), \\
& =P_{h} D_{x \mp}^{\alpha} f(x)+O\left(h^{3}\right) .
\end{aligned}
$$

This approach is analogous to that of Hao et al.[3], where they used convex combinations of three shifted operators from the first order Grünwald approximations $W_{1}(z)$ resulting in a different approximation operator. In our case, the same second order approximation operator $\Delta_{h \mp, 1,2}^{\alpha}$ is used to obtain a third order accuracy for a preconditioned operator $\mathrm{P}_{\mathrm{h}} \mathrm{D}_{\chi \mp}^{\alpha}$. 


\section{Fractional diffusion equation}

We consider the numerical approximation of the space fractional diffusion problem in the domain $[a, b] \times[0, T]$ :

$$
\begin{aligned}
\frac{\partial}{\partial t} u(x, t) & =K_{1} D_{x-}^{\alpha} u(x, t)+K_{2} D_{x+}^{\alpha} u(x, t)+f(x, t), \\
u(x, 0) & =s_{0}(x) ; \\
u(a, t) & =\phi_{1}(t), u(b, t)=\phi_{2}(t),
\end{aligned}
$$

where $\mathfrak{u}(x . t)$ is the unknown function to be determined; $K_{1}, K_{2}$ are nonnegative constant diffusion coefficients with $\mathrm{K}_{1}+\mathrm{K}_{2} \neq 0$, i.e, not both are simultaneously zero, and $f(x, t)$ is a known source term. The boundary conditions are set as follows: For $i=1,2$, if $\boldsymbol{K}_{\boldsymbol{i}} \neq 0$, then $\phi_{\boldsymbol{i}}(\mathrm{t}) \equiv 0$. We assume that the diffusion problem (11) has a unique solution.

To obtain a third order approximation with the use of (7), we first pre-multiply the equation (11) by $P_{h}$ in equation (9) to get

$$
P_{h} \frac{\partial u(x, t)}{\partial t}=K_{1} P_{h} D_{x-}^{\alpha} u(x, t)+K_{2} P_{h} D_{x+}^{\alpha} u(x, t)+P_{h} f(x, t) .
$$

The preconditioned left and right FDs are then approximated by $\Delta_{h \mp, 1,2}^{\alpha} \mathfrak{u}(x, t)$ respectively with order 3 accuracy established in Subsection 2.1.

$$
\begin{aligned}
P_{h} \frac{\partial u(x, t)}{\partial t} & =K_{1} \Delta_{h-, 1,2}^{\alpha} u(x, t)+K_{2} \Delta_{h+, 1,2}^{\alpha} u(x, t)+P_{h} f(x, t)+O\left(h^{3}\right), \\
& =: B_{h} u(x, t)+P_{h} f(x, t)+O\left(h^{3}\right) .
\end{aligned}
$$

Approximating the time derivative with the second order $\mathrm{C}-\mathrm{N}$ scheme gives $P_{h} \frac{1}{\tau}(u(x, t+\tau)-u(x, t))=\frac{1}{2} B_{h}(u(x, t)+u(x, t+\tau))+P_{h} f\left(x, t+\frac{\tau}{2}\right)+O\left(h^{3}+\tau^{2}\right)$.

Discretise the domain $[a, b] \times[0, T]$ with size $N \times M$ by choosing step sizes $h=(b-a) / N, \tau=T / M$ and grid points $\left(x_{i}, t_{m}\right)$, where $x_{i}=a+i h$, $t_{m}=m \tau, 0 \leqslant i \leqslant N, \quad 0 \leqslant m \leqslant M$. 
Denoting $u_{i}^{m}=u\left(x_{i}, t_{m}\right), t_{m+1 / 2}=\frac{1}{2}\left(t_{m+1}+t_{m}\right)$ and $f_{i}^{m+1 / 2}=f\left(x_{i}, t_{m+1 / 2}\right)$, the $\mathrm{C}-\mathrm{N}$ scheme in matrix form with $\mathrm{U}^{\mathrm{m}}=\left[\mathfrak{u}_{0}^{\mathrm{m}}, \mathfrak{u}_{1}^{\mathfrak{m}}, \cdots, \mathfrak{u}_{\mathrm{N}}^{\mathfrak{m}}\right]^{\top}, \mathrm{F}^{\mathrm{m}+1 / 2}=$ $\left[\mathrm{f}_{0}^{\mathrm{m}+1 / 2}, \mathrm{f}_{1}^{\mathrm{m}+1 / 2}, \cdots, \mathrm{f}_{\mathrm{N}}^{\mathrm{m}+1 / 2}\right]^{\mathrm{T}}$, after re-arranging, reads,

$$
\left(P_{\alpha}-B_{\alpha}\right) U^{m+1}=\left(P_{\alpha}+B_{\alpha}\right) U^{m}+\tau P_{\alpha} F^{m+1 / 2}+O\left(\tau\left(h^{3}+\tau^{2}\right)\right),
$$

where $P_{\alpha}=\operatorname{Tri}\left[a_{2}, 1-2 a_{2}, a_{2}\right]$ is a tridiagonal matrix of size $(N+1)$ corresponding to $P_{h}, B_{\alpha}=\frac{\tau}{2}\left(K_{1} A_{1,2}+K_{2} A_{1,2}^{\top}\right)$, where $A_{1,2}$ and $A_{1,2}^{\top}$ are toeplitz matrices corresponding to the left and right approximation operators $\Delta_{h \mp, 1,2}^{\alpha}$ respectively with $A_{1,2}=\left[w_{i-j+1,1}\right]_{(\mathrm{N}+1) \times(\mathrm{N}+1)}$. Imposing boundary conditions and discarding the remainder term, we have

$$
\left(\hat{\mathrm{P}}_{\alpha}-\hat{\mathrm{B}}_{\alpha}\right) \hat{\mathrm{U}}^{\mathrm{m}+1}=\left(\hat{\mathrm{P}}_{\alpha}+\hat{\mathrm{B}}_{\alpha}\right) \hat{\mathrm{U}}^{\mathrm{m}}+\tau \hat{\mathrm{P}}_{\alpha} \hat{\mathrm{F}}^{\mathrm{m}+1 / 2}+\hat{\mathbf{b}}^{\mathrm{m}}, \mathrm{m}=0,1, \cdots, M-1,
$$

where the hatted matrices and vectors are the $(\mathrm{N}-1)$ sized reduced forms by deleting the first and the last rows and/or columns of their counterparts in (12). The vector $\hat{\mathbf{b}}^{\mathrm{m}}$ corresponds to the boundary conditions given by $\hat{\mathbf{b}}^{\mathrm{m}}=\left(\mathrm{P}_{\alpha}+\mathrm{B}_{\alpha}\right)_{0} \phi_{1}^{\mathrm{m}}+\left(\mathrm{P}_{\alpha}+\mathrm{B}_{\alpha}\right)_{\mathrm{N}} \phi_{2}^{\mathrm{m}}-\left(\mathrm{P}_{\alpha}-\mathrm{B}_{\alpha}\right)_{0} \phi_{1}^{\mathrm{m}+1}-\left(\mathrm{P}_{\alpha}-\mathrm{B}_{\alpha}\right)_{\mathrm{N}} \phi_{2}^{\mathrm{m}+1}$, where the subscripts 0 and $N$ denote the first and last columns of relevant matrices reduced to size $(\mathrm{N}-1)$ as above.

\subsection{Stability and convergence}

For stability of the C-N scheme (12), we closely follow the analysis in [3] and present some required results.

Let $V_{h}=\left\{v \mid v=\left(v_{0}, v_{1}, \cdots, v_{N}\right), v_{i} \in \mathbb{R}, v_{0}=v_{N}=0\right\}$ be the space of grid functions in the computational domain in space interval $[a, b]$. 
For any $u, v \in V_{h}$, define the discrete inner products and norms as

$$
\begin{aligned}
(\mathfrak{u}, v) & =\mathrm{h} \sum_{i=1}^{\mathrm{N}-1} \mathfrak{u}_{\mathrm{i}} v_{\mathrm{i}}, & \|\mathfrak{u}\|=\sqrt{(\mathfrak{u}, \mathfrak{u}),} \\
\left\langle\delta_{\mathrm{h}} \mathrm{u}, \delta_{\mathrm{h}} v\right\rangle & =\mathrm{h} \sum_{i=1}^{\mathrm{N}-1}\left(\delta_{\mathrm{h}} \mathrm{u}_{\mathrm{i}-1 / 2}\right)\left(\delta_{\mathrm{h}} v_{\mathrm{i}-1 / 2}\right) \text { and } & |\mathfrak{u}|_{1}=\sqrt{\left\langle\delta_{\mathrm{h}} \mathfrak{u}, \delta_{\mathrm{h}} \mathfrak{u}\right\rangle} .
\end{aligned}
$$

Then, we have the following lemmas [7].

Lemma 4. Let $\left\{\mathrm{t}_{\mp \mathrm{k}}\right\}_{\mathrm{k}=0}^{\infty}$ be a double sided real sequence such that (i) $\mathrm{t}_{\mathrm{k}}+\mathrm{t}_{-\mathrm{k}} \geqslant 0$ for $\mathrm{k} \neq 0$, (ii) $\sum_{\mathrm{j}=-\mathrm{N}}^{\mathrm{N}} \mathrm{t}_{\mathrm{j}} \leqslant 0$ for $\mathrm{N} \geqslant 0$. Then, the toeplitz matrices $\mathrm{T}_{\mathrm{N}}=\left[\mathrm{t}_{\mathrm{i}-\mathrm{j}}\right]$ of size $(\mathrm{N}+1)$ are negative definite.

Lemma 5. The operators $\Delta_{\mathrm{h \mp}, 1,2}^{\alpha}$ are negative definite for $1 \leqslant \alpha \leqslant 2$ and hence $\Delta_{2}^{\alpha}=\mathrm{K}_{1} \Delta_{\mathrm{h}-, 1,2}^{\alpha}+\mathrm{K}_{2} \Delta_{\mathrm{h}+1,2}^{\alpha}$ is negative definite.

In addition, we have

Lemma 6. The operator $\mathrm{P}_{\mathrm{h}}$ is self-adjoint and $\left(\mathrm{P}_{\mathrm{h}} \mathrm{u}, \mathfrak{u}\right)=:\|\mathfrak{u}\|_{\mathrm{P}}^{2}$ satisfies $\frac{1}{5}\|\mathrm{u}\|^{2}<\|\mathrm{u}\|_{\mathrm{P}}^{2} \leqslant\|\mathrm{u}\|^{2}$.

Proof: $\quad\left(\mathrm{P}_{\mathrm{h}} \mathrm{u}, v\right)=\left(\left(\mathrm{I}+\mathrm{h}^{2} \mathrm{a}_{2} \delta_{\mathrm{h}}^{2}\right) \mathrm{u}, v\right)=(\mathrm{u}, v)+\mathrm{h}^{2} \mathrm{a}_{2}\left(\delta_{\mathrm{h}}^{2} \mathrm{u}, v\right)=(\mathrm{u}, v)-$ $h^{2} a_{2}\left(\delta_{h} u, \delta_{h} v\right)=(u, v)+h^{2} a_{2}\left(u, \delta_{h}^{2} v\right)=\left(u,\left(I+h^{2} a_{2} \delta_{h}^{2}\right) v\right)=\left(u, P_{h} v\right)$.

Moreover, for $1 \leqslant \alpha \leqslant 2$, we easily see that, with $r=1, a_{2}=-\frac{\alpha}{3}+1-\frac{1}{2 \alpha}$ satisfies $\frac{1}{12} \leqslant a_{2} \leqslant 1-\frac{\sqrt{6}}{3}<\frac{1}{5}$.

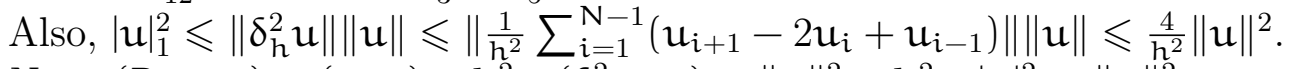

Now, $\left(P_{h} \mathfrak{u}, \mathfrak{u}\right)=(\mathfrak{u}, \mathfrak{u})+h^{2} a_{2}\left(\delta_{h}^{2} \mathfrak{u}, \mathfrak{u}\right)=\|\mathfrak{u}\|^{2}-h^{2} a_{2}|\mathfrak{u}|_{1}^{2} \leqslant\|\mathfrak{u}\|^{2}$.

Again, $\left(P_{h} u, u\right)=\|\mathfrak{u}\|^{2}-h^{2} a_{2}|\mathfrak{u}|_{1}^{2}>\|\mathfrak{u}\|^{2}-\frac{4}{5}\|\mathfrak{u}\|^{2}=\frac{1}{5}\|\mathfrak{u}\|^{2}$. 
Theorem 7. Let $v^{\mathrm{m}}=\left(v_{1}^{\mathrm{m}}, v_{2}^{\mathrm{m}}, \cdots, v_{\mathrm{N}-1}^{\mathrm{m}}\right)$ be the solution of the problem

$$
\begin{gathered}
\mathrm{P}_{\mathrm{h}} \delta_{\mathrm{t}} v_{\mathrm{i}}^{\mathrm{m}+1 / 2}-\Delta_{2}^{\alpha} v_{i}^{\mathrm{m}+1 / 2}=S_{i}^{\mathrm{m}}, \quad 1 \leqslant i \leqslant \mathrm{~N}-1, \quad 0 \leqslant \mathrm{~m} \leqslant \mathrm{M}-1, \\
v_{0}^{\mathrm{m}}=0, \quad v_{\mathrm{N}}^{\mathrm{m}}=0, \quad v_{i}^{0}=v^{0}\left(x_{i}\right), \quad 0 \leqslant i \leqslant \mathrm{~N} .
\end{gathered}
$$

Then, $\left\|v^{\mathrm{m}}\right\| \leqslant \sqrt{5}\left(\left\|v^{0}\right\|+\sqrt{5} \tau \sum_{\mathrm{l}=0}^{\mathrm{m}-1}\left\|\mathrm{~S}^{\mathrm{l}}\right\|\right)$, where $\mathrm{S}^{\mathrm{l}}=\left[\mathrm{S}_{1}^{\mathrm{l}}, \mathrm{S}_{2}^{\mathrm{l}}, \cdots, \mathrm{S}_{\mathrm{N}-1}^{\mathrm{l}}\right]^{\mathrm{T}}$.

Proof: Applying inner product on (14) with $v^{\mathrm{m}+1 / 2}$ and, from Lemma 5, using $\left(\Delta_{2}^{\alpha} v^{m+1 / 2}, v^{m+1 / 2}\right) \leqslant 0$ gives

$$
\left(\mathrm{P}_{\mathrm{h}} \delta_{\mathrm{t}} v^{\mathrm{m}+1 / 2}, v^{\mathrm{m}+1 / 2}\right)=\left(\Delta_{2}^{\alpha} v^{\mathrm{m}+1 / 2}, v^{\mathrm{m}+1 / 2}\right)+\left(\mathrm{S}^{\mathrm{m}}, v^{\mathrm{m}+1 / 2}\right) \leqslant\left(\mathrm{S}^{\mathrm{m}}, v^{\mathrm{m}+1 / 2}\right) .
$$

Also,

$$
\begin{aligned}
\left(\mathrm{P}_{\mathrm{h}} \delta_{\mathrm{t}} v^{\mathrm{m}+1 / 2}, v^{\mathrm{m}+1 / 2}\right) & =\left(\mathrm{P}_{\mathrm{h}} \frac{1}{\tau}\left(v^{\mathrm{m}+1}-v^{\mathrm{m}}\right), \frac{1}{2}\left(v^{\mathrm{m}+1}+v^{\mathrm{m}}\right)\right), \\
& =\frac{1}{2 \tau}\left(\left\|v^{\mathrm{m}+1}\right\|_{\mathrm{P}}^{2}-\left\|v^{\mathrm{m}}\right\|_{\mathrm{P}}^{2}\right), \\
& \leqslant\left(S^{\mathrm{m}}, v^{\mathrm{m}+1 / 2}\right) \leqslant\left\|S^{\mathrm{m}}\right\|\left\|v^{\mathrm{m}+1 / 2}\right\|, \\
& \leqslant \sqrt{5}\left\|S^{\mathrm{m}}\right\|\left\|v^{\mathrm{m}+1 / 2}\right\|_{\mathrm{P}}, \\
& \leqslant \frac{\sqrt{5}}{2}\left\|S^{\mathrm{m}}\right\|\left(\left\|v^{\mathrm{m}+1}\right\|_{\mathrm{P}}+\left\|v^{\mathrm{m}}\right\|_{\mathrm{P}}\right) .
\end{aligned}
$$


Theorem 8. The $\mathrm{C}-\mathrm{N}$ scheme (12) is unconditionally stable for $1 \leqslant \alpha \leqslant 2$. Theorem 9. The approximate solutions of the $\mathrm{C}-\mathrm{N}$ scheme (12) with the given initial and boundary conditions are convergent for $1 \leqslant \alpha \leqslant 2$.

Proof: Let $e^{m}=u^{m}-\hat{U}^{m}$ be the error vector of the solutions, where $u^{m}, \hat{U}^{m}$ are the exact and approximate solution vectors of the diffusion problem (11). Then the error of the internal grid values $e^{m}$ satisfies the system

$\mathrm{P}_{\mathrm{h}} \delta_{\mathrm{t}} e_{i}^{\mathrm{m}+1 / 2}-\Delta_{2}^{\alpha} e_{i}^{\mathrm{m}+1 / 2}=R_{i}^{\mathrm{m}}, \quad 1 \leqslant i \leqslant N-1, \quad 0 \leqslant \mathrm{~m} \leqslant \mathrm{M}-1$, $e_{0}^{\mathfrak{m}}=0, \quad e_{M}^{\mathfrak{m}}=0 \quad e_{i}^{0}=0, \quad 0 \leqslant i \leqslant N$.

Theorem 7 then gives the estimate $\left\|e^{m}\right\| \leqslant 5 \tau \sum_{l=0}^{m-1}\left\|R^{l}\right\| \leqslant 5 c \tau N\left(\tau^{2}+h^{3}\right)$. The convergence is then established as $h, \tau \rightarrow 0$.

\section{Numerical results}

We test our order 3 approximation through a steady state problem and a time dependent diffusion problem for a range of exact solutions that are sufficiently smooth to realise the order 3 approximations and that are not sufficiently smooth to see if any reduction in order mentioned in [1] is detected.

Example 1. Consider the steady state fractional diffusion problem with left fractional derivative $\mathrm{D}_{\mathrm{x}-}^{\alpha} \mathfrak{u}(\mathrm{x})=\chi^{\gamma} \Gamma(\gamma+\alpha+1) / \Gamma(\gamma+1), \quad \mathfrak{u}(0)=0$, with exact solution $u(x)=x^{\gamma+\alpha}, \quad 0 \leqslant x \leqslant 1, \quad 0 \leqslant \gamma \leqslant 6$ and $1 \leqslant \alpha \leqslant 2$. Note that $\mathrm{D}^{\beta} \mathrm{u}(\mathrm{x}) \in \mathrm{L}_{1}[0,1]$ for $\beta \leqslant \gamma+\alpha+1$.

This steady state test problem was approximated by $\mathrm{C}-\mathrm{N}$ numerical scheme (13) for 21 selected equi-spaced discrete power values in the range $0 \leqslant \gamma \leqslant 6$. The maximum error $\mathrm{E}_{N}=\|\mathrm{u}-\mathrm{U}\|_{\infty}$ and the orders of convergence were computed for values of $\alpha=1.1,1.5$ and 1.8 with space partition sizes $\mathrm{N}=256$ and 512. Figure 1 shows the plots of the orders of convergence against the 
power $\gamma$. This indicates that the convergence order is achieved only for sufficiently smooth functions and is reduced for less-smooth functions. This phenomenon requires an analogous analysis as given in [1] with our third order approximation.

Example 2. Consider the test example for the fractional diffusion problem (11). Let $\mathrm{G}(\mathrm{x}, \mathrm{m}, \alpha)=\frac{\Gamma(\mathrm{m}+1)}{\Gamma(\mathrm{n}+1-\alpha)}\left(\mathrm{x}^{\mathrm{m}-\alpha}+(1-\chi)^{\mathrm{m}-\alpha}\right)$ and $\mathrm{s}_{0}(x)=\chi^{5}(1-\chi)^{5}$ with the following data:

Diffusion coefficients: $\quad \mathrm{K}_{1}=1, \mathrm{~K}_{2}=1$,

Source function: $\quad f(x, t)=-e^{-t}\left(s_{0}(x)+G(x, 5, \alpha)-5 G(x, 6, \alpha)+\right.$ $10 \mathrm{G}(x, 7, \alpha)-10 \mathrm{G}(x, 8, \alpha)+5 \mathrm{G}(x, 9, \alpha)-\mathrm{G}(x, 10, \alpha))$,

Initial condition: $\mathfrak{u}(x, 0)=s_{0}(x)$,

Boundary conditions: $\quad \mathfrak{u}(0, \boldsymbol{t})=0, \quad \mathfrak{u}(1, \mathfrak{t})=0$.

Exact Solution: $u(x, t)=s_{0}(x) e^{-t}$.

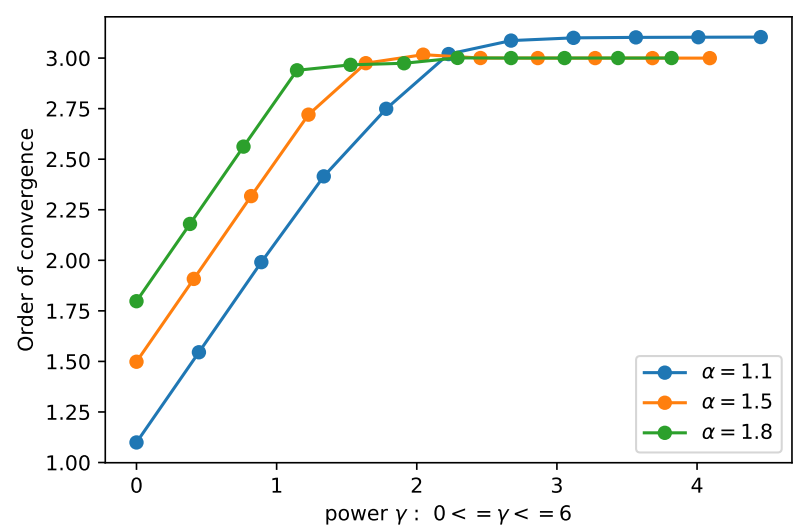

Figure 1: Convergence orders for Example 1 
The space domain is partitioned with size $\mathrm{N}$ and the time domain is partitioned with size $M=\left[\mathrm{N}^{3 / 2}\right]+1$. The choice of time partition is to realise the order 3 with the $\mathrm{C}-\mathrm{N}$ scheme of original order 2 . The order $p$ is computed by the formula $p_{n}=\log \left(\left\|E^{n}\right\|_{\infty} /\left\|E^{n-1}\right\|_{\infty}\right) / \log 2$ for $n=4,5, \cdots, 9$, where the error $E^{n}=u^{n}-U^{n}$.

The test results listed in Tables 1 show that the order 3 convergence is achieved for the sufficiently smooth exact solution.

\begin{tabular}{rrllllll}
\hline & & $\alpha=1.1$ & & $\alpha=1.5$ & & $\alpha=1.9$ & \\
\hline $\mathrm{N}=1 / \mathrm{h}$ & $\mathrm{M}=1 / \tau$ & $\left\|\mathrm{E}^{\mathfrak{n}}\right\|_{\infty}$ & Order & $\left\|\mathrm{E}^{\mathfrak{n}}\right\|_{\infty}$ & Order & $\left\|\mathrm{E}^{\mathfrak{n}}\right\|_{\infty}$ & Order \\
\hline 16 & 65 & $1.9 \mathrm{e}-06$ & - & $7.2 \mathrm{e}-07$ & - & $2.9 \mathrm{e}-08$ & - \\
32 & 182 & $2.4 \mathrm{e}-07$ & 2.97 & $9.1 \mathrm{e}-08$ & 2.99 & $2.7 \mathrm{e}-09$ & 3.40 \\
64 & 513 & $3.1 \mathrm{e}-08$ & 2.99 & $1.1 \mathrm{e}-08$ & 3.00 & $5.3 \mathrm{e}-10$ & 2.35 \\
128 & 1449 & $3.9 \mathrm{e}-09$ & 2.99 & $1.4 \mathrm{e}-09$ & 3.00 & $7.9 \mathrm{e}-11$ & 2.76 \\
256 & 4097 & $4.9 \mathrm{e}-10$ & 3.00 & $1.7 \mathrm{e}-10$ & 3.00 & $1.0 \mathrm{e}-11$ & 2.90 \\
512 & 11586 & $6.1 \mathrm{e}-11$ & 3.00 & $2.2 \mathrm{e}-11$ & 3.00 & $1.3 \mathrm{e}-12$ & 2.95 \\
\hline
\end{tabular}

Table 1: Order 3 convergence of $\mathrm{C}-\mathrm{N}$ type scheme

\section{Conclusion}

A new third order approximation for fractional derivative was developed using a second order approximation with a pre-conditioner operator. A C-N numerical scheme for this approximation to solve FDE was devised with proof of stability and convergence. The theoretical predictions were tested and verified for fractional diffusion problems through examples with sufficiently smooth solutions and less-smooth solutions

Acknowledgement We are grateful to one of the referees for suggesting some references which are very much close to our work that improved the presentation of this work. This research was supported by Sultan Qaboos University Internal Grant No. IG/SCI/DOMAS/16/10. 


\section{References}

[1] Boris Baeumer, Mihály Kovács, and Harish Sankaranarayanan. Higher order grünwald approximations of fractional derivatives and fractional powers of operators. Transactions of the American Mathematical Society, 367(2):813-834, 2015. doi:10.1090/S0002-9947-2014-05887-X C257, C265, C266

[2] E. Barkai, R. Metzler, and J. Klafter. From continuous time random walks to the fractional fokker-planck equation. Physical Review E, 61(1):132, 2000. doi:10.1103/PhysRevE.61.132 C255

[3] Z. Hao, Z. Sun, and W. Cao. A fourth-order approximation of fractional derivatives with its applications. Journal of Computational Physics, 281:787-805, 2015. doi:10.1016/j.jcp.2014.10.053 C260, C262

[4] Ch Lubich. Discretized fractional calculus. SIAM Journal on Mathematical Analysis, 17(3):704-719, 1986. doi:10.1137/0517050 C256, $\mathrm{C} 259$

[5] M. M. Meerschaert and C. Tadjeran. Finite difference approximations for fractional advection-dispersion flow equations. Journal of Computational and Applied Mathematics, 172(1):65-77, 2004. doi:10.1016/j.cam.2004.01.033 C256

[6] H. M. Nasir, B. L. K. Gunawardana, and H. M. N. P. Aberathna. A second order finite difference approximation for the fractional diffusion equation. International Journal of Applied Physics and Mathematics, 3(4):237, 2013. doi:10.7763/IJAPM.2013.V3.212 C257

[7] H. M. Nasir and K. Nafa. A new second order approximation for fractional derivatives with applications. SQU Journal of Science, 23(1):43-55, 2018. doi:10.24200/squjs.vol23iss1pp43-55 C257, C258, C259, C263 
[8] W. Tian, H. Zhou, and W. Deng. A class of second order difference approximations for solving space fractional diffusion equations. Mathematics of Computation, 84(294):1703-1727, 2015. doi:10.1090/S0025-5718-2015-02917-2 C257

[9] Y. Yu, W. Deng, and Y. Wu. Fourth order quasi-compact difference schemes for (tempered) space fractional diffusion equations. arXiv preprint arXiv:1408.6364, 2014. doi:10.4310/CMS.2017.v15.n5.a1 C257

[10] Y. Yu, W. Deng, Y. Wu, and J. Wu. Third order difference schemes (without using points outside of the domain) for one sided space tempered fractional partial differential equations. Applied Numerical Mathematics, 112:126-145, 2017. doi:10.1016/j.apnum.2016.10.01 C257

[11] L. Zhao and W. Deng. A series of high-order quasi-compact schemes for space fractional diffusion equations based on the superconvergent approximations for fractional derivatives. Numerical Methods for Partial Differential Equations, 31(5):1345-1381, 2015. doi:10.1002/num.21947 $\mathrm{C} 257$

\section{Author addresses}

1. HM Nasir, FracDiff Research Group, Department of Mathematics, Sultan Qaboos University, P.O.Box:36, Al-Khoud, P.C : 123, Muscat, Sultanate of OMAn.

mailto:nasirh@squ.edu.om, nasirhm11@yahoo.com orcid:0000-0002-4476-7786

2. K Nafa, Department of Mathematics, Sultan Qaboos University, P.O.Box:36, Al-Khoud, P.C : 123, Muscat, Sultanate of Oman. mailto:nkamelesqu.edu.om 